Preface

\title{
Neurocritical Care and Emergency Neurology: Current Evidence and Consensus Practice
}

\author{
David Y. Hwang, MD, FNCS ${ }^{1,2}$ David M. Greer, MD, MA, FCCM, FAHA, FNCS, \\ FAAN, FANA ${ }^{3}$ \\ ${ }^{1}$ Division of Neurocritical Care and Emergency Neurology, \\ Department of Neurology, Yale School of Medicine, \\ New Haven, Connecticut \\ ${ }^{2}$ Center for Neuroepidemiology and Clinical Neurological Research, \\ Yale School of Medicine, New Haven, Connecticut \\ ${ }^{3}$ Department of Neurology, Boston University School of Medicine, \\ Boston, Massachusetts
}

Semin Respir Crit Care Med 2017;38:711-712.

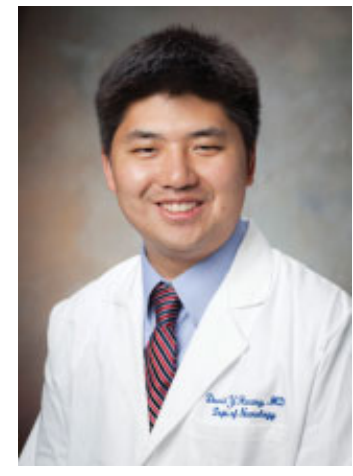

David Y. Hwang, MD, FNCS

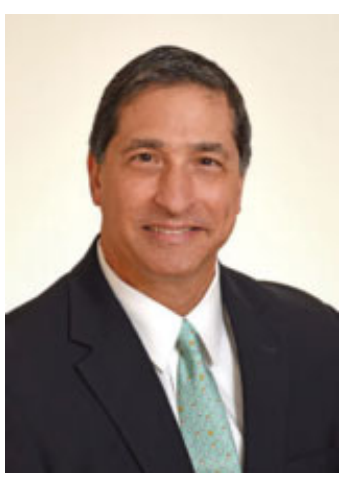
novel oral anticoagulants.

The following three articles in this David M. Greer, MD, MA, issue delve deeply into major neurovas- FCCM, FAHA, FNCS, FAAN, cular diseases cared for in neuro-ICUs: FANA hemispheric ischemic stroke, spontaneous intraventricular hemorrhage (IVH), and aneurysmal subarachnoid hemorrhage (aSAH). Landreneau and Sheth discuss the state of the evidence for decompressive craniectomy for patients with large middle cerebral artery infarction. Decisions to pursue surgery for these patients are made on a case-by-case basis, but recent data regarding outcomes in older patients may help inform these discussions. Similarly, relatively recent trial data may help guide clinicians who may be considering use of intraventricular fibrinolysis via external ventricular drain(s) to treat patients presenting with spontaneous IVH; Abdelmalik and Ziai discuss the general approach to the subset of ICH patients with IVH and the further subset who might benefit from a trial of intraventricular tPA. Admittedly, scientifically proven advancements in the prevention of delayed cerebral ischemia seen in patients with aSAH have been hard to come by, as enteral nimodipine remains the only proven medical therapy to impact clinical outcome in these patients. However, Diringer and Zazulia summarize what has been tried to date and
Address for correspondence David Y. Hwang, MD, FNCS, Division of Neurocritical Care and Emergency Neurology, Department of Neurology, Yale School of Medicine, P.O. Box 208018, New Haven, CT 06520 (e-mail: david.hwang@yale.edu).
Issue Theme Advancements in Neurocritical Care and Emergency Neurology; Guest Editors: David Y. Hwang, MD, FNCS, and David M. Greer, MD, MA, FCCM, FAHA, FNCS, FAAN, FANA
Copyright $₫ 2017$ by Thieme Medical Publishers, Inc., 333 Seventh Avenue, New York, NY 10001, USA.

Tel: +1(212) 584-4662.
DOI https://doi.org/ 10.1055/s-0037-1608797. ISSN 1069-3424. 
promising new therapies being tested, including the intraventricular administration of nimodipine.

Two additional articles focus on classic neuro-ICU diseases: traumatic brain injury (TBI) and hypoxic-ischemic injury following cardiac arrest. Neurocritical care management of severe TBI is an expansive topic; Chiu and Hinson specifically focus on summarizing the state of the evidence regarding the use of therapeutic hypothermia for these patients. While cooling TBI patients can reduce intracranial pressure (ICP), recent data have called into question its impact on longer term clinical outcomes. Research on neuroprognostication following cardiac arrest has been very robust, with impactful articles on this topic being published on a near-monthly basis. Cronberg provides an overall summary of this topic with a specific focus on the limitations of outcome measures that have served as the basis for most studies to date, as well as the need to better understand the cognitive outcomes among cardiac arrest survivors.

Advancements in technology have impacted the neurocritical care specialist's ability to monitor the brain across different disease types, in addition to reliance on serial neurologic examinations and neuroimaging. Gandee and Miller summarize options available for what has been termed "multimodality monitoring" in neurocritical care. In addition to the traditional measurement of ICP and cerebral perfusion pressure, these include transcranial Doppler, tissue oxygen monitoring, and cerebral microdialysis techniques. In particular, the ability to obtain continuous electroencephalograms (cEEGs) in neurocritically ill patients gives clinicians a tremendous amount of data but raises issues in terms of how to manage patients whose cEEG patterns may not show frank seizures but are not "normal" either. Cormier, Maciel, and Gilmore summarize a clinical approach to what has been termed the "ictal-interictal continuum" among epileptologists and neurointensivists.
This issue contains articles on two diseases which are seen with less frequency than stroke, TBI, and cardiac arrest but which deserve mention because of the therapeutic conundrums that they present even to specialists. Rubin et al review the topic of autoimmune encephalitis in neuro-ICUs. These conditions can be difficult to diagnosis, and the authors advocate for early intervention with autoimmune therapy, as favorable outcomes for patients with these diseases have been seen with aggressive empiric management. For patients with acute liver failure, cerebral edema is a well-described complication, but significant practice variability exists over how best to monitor and treat this complication. Kok and Karvellas discuss methods of lowering ICP for these patients.

Timing of extubation and obtaining appropriate diagnostic imaging are issues relevant to nearly all ICU patients, but special considerations exist for the neuro-ICU population. Bösel addresses predictors of extubation success and failure in patients who may have normal pulmonary mechanics but severe brain injury and discusses a stepwise approach to extubation decision making in the neuro-ICUs. The final article of this issue by Williamson, Morgan, and Klein is a comprehensive overview of neuroimaging in neurocritical care. Specific situations in which one might truly have a need for magnetic resonance imaging over CT are covered in detail, and considerations regarding safe patient transport to scanners are addressed.

We thank each of our author contributors very much for the work they have put into this issue and Dr. Joseph Lynch for our opportunity to guest edit. Our hopes are that this broad issue will be of interest both to the communities of general critical care clinicians and neurocritical care specialists alike and that, while it is the first issue of this journal to be focused on the neuro-ICU, it will not be the last.

\section{Conflict of Interest and Source of Funding}

No funding or relevant conflict of interest reported. 